\title{
Comparison and Validation of FLUKA and HZETRN as Tools for Investigating the Secondary Neutron Production in Large Space Vehicles
}

\author{
Kristina Rojdev ${ }^{1}$, Steve Koontz ${ }^{2}$ and Brandon Reddell ${ }^{3}$ \\ NASA Johnson Space Center, Houston, TX, 77058 \\ William Atwell ${ }^{4}$ \\ Retired Boeing Technical Fellow, Houston, TX, 77058 \\ and \\ Paul Boeder ${ }^{5}$ \\ Boeing Research and Technology, Houston, TX, 77058
}

\begin{abstract}
NASA's exploration goals are focused on deep space travel and Mars surface operations. To accomplish these goals, large structures will be necessary to transport crew and logistics in the initial stages, and NASA will need to keep the crew and the vehicle safe during transport and any surface activities. One of the major challenges of deep space travel is the space radiation environment and its impacts on the crew, the electronics, and the vehicle materials. The primary radiation from the sun (solar particle events) and from outside the solar system (galactic cosmic rays) interact with materials of the vehicle. These interactions lead to some of the primary radiation being absorbed, being modified, or producing secondary radiation (primarily neutrons). With all vehicles, the high energy primary radiation is of most concern. However, with larger vehicles that have large shielding masses, there is more opportunity for secondary radiation production, and this secondary radiation can be significant enough to cause concern. When considering surface operations, there is also a secondary radiation source from the surface of the planet, known as albedo, with neutrons being one of the most significant species. Given new vehicle designs for deep space and Mars missions, the secondary radiation environment and the implications of that environment is currently not well understood. Thus, several studies are necessary to fill the knowledge gaps of this secondary radiation environment. In this paper, we put forth the initial steps to increasing our understanding of neutron production from large vehicles by comparing the neutron production resulting from our radiation transport codes and providing a preliminary validation of our results against flight data. This paper will review the details of these results and discuss the finer points of the analysis.
\end{abstract}

\section{Nomenclature}

GCR = galactic cosmic radiation

SPE $\quad=$ solar particle event

\section{Introduction}

A S NASA plans to venture into deep space and eventually Mars, vehicle designs and configurations are increasing in size to accommodate the additional supplies needed for longer duration missions ${ }^{1-2}$. On the one hand, an increase in vehicle thickness provides some shielding to crew and electronics against lower energy primary radiation

${ }^{1}$ Aerospace Engineer, Systems Engineering \& Test Branch, 2101 NASA Parkway/MC:EA351, AIAA Member.

${ }^{2}$ International Space Station System Manager for Space Environments, 2101 NASA Parkway/MC:ES4, AIAA Member.

${ }^{3}$ Avionics Systems Division, 2101 NASA Parkway/MC:EV5.

${ }^{4}$ Retired, 16623 Park Green Way, AIAA Associate Fellow.

${ }^{5}$ Aerospace Engineer, Space Environments, 13100 Space Center Blvd./HB3-20. 
resulting from solar particle events. However, larger vehicle thicknesses also provide more material for high-energy particles, such as galactic cosmic radiation, to interact and produce secondary neutrons. The secondary neutron environment from these new vehicle configurations is not well understood and the implications of this neutron environment could be cause for concern to crew, electronics, and materials. In this paper, we will take a first step at examining the secondary neutron production from various vehicle thicknesses using two different radiation transport codes. We will compare our simulated results with flight data to validate our calculations. Finally, we will discuss the implications of these results and the next steps necessary to further this investigation.

\section{Background}

\section{A. Radiation Environment}

There are two types of radiation sources that will be discussed. The first is primary radiation, which is a radiation source that has not interacted with any other material. The second source is secondary radiation and is due to primary radiation interacting with a medium to create secondary products such as neutrons.

\section{Primary Radiation}

Solar particle events (SPEs) and galactic cosmic radiation (GCR) are the two major sources of primary space radiation for deep space missions. A secondary source, discussed in the next section, of space radiation (mostly neutrons) occurs from nuclear reactions when the incident SPE and/or GCR particles penetrate the bulk shielding of the spacecraft and other interior components, including the human body and avionics. These primary radiation sources are high energy protons and stripped nuclei up to iron $(\mathrm{Fe})$. For heavily-shielded spacecraft, only extremely energetic SPEs are a concern, such as Ground Level Events (GLEs) discussed in ref 3.

Examples of two GLEs are shown in Figure 1, which shows the integral and differential energy spectra for the series of SPEs that occurred during 19-24 October 1989 and the "Bastille Day" event that occurred on 14 July 2000.

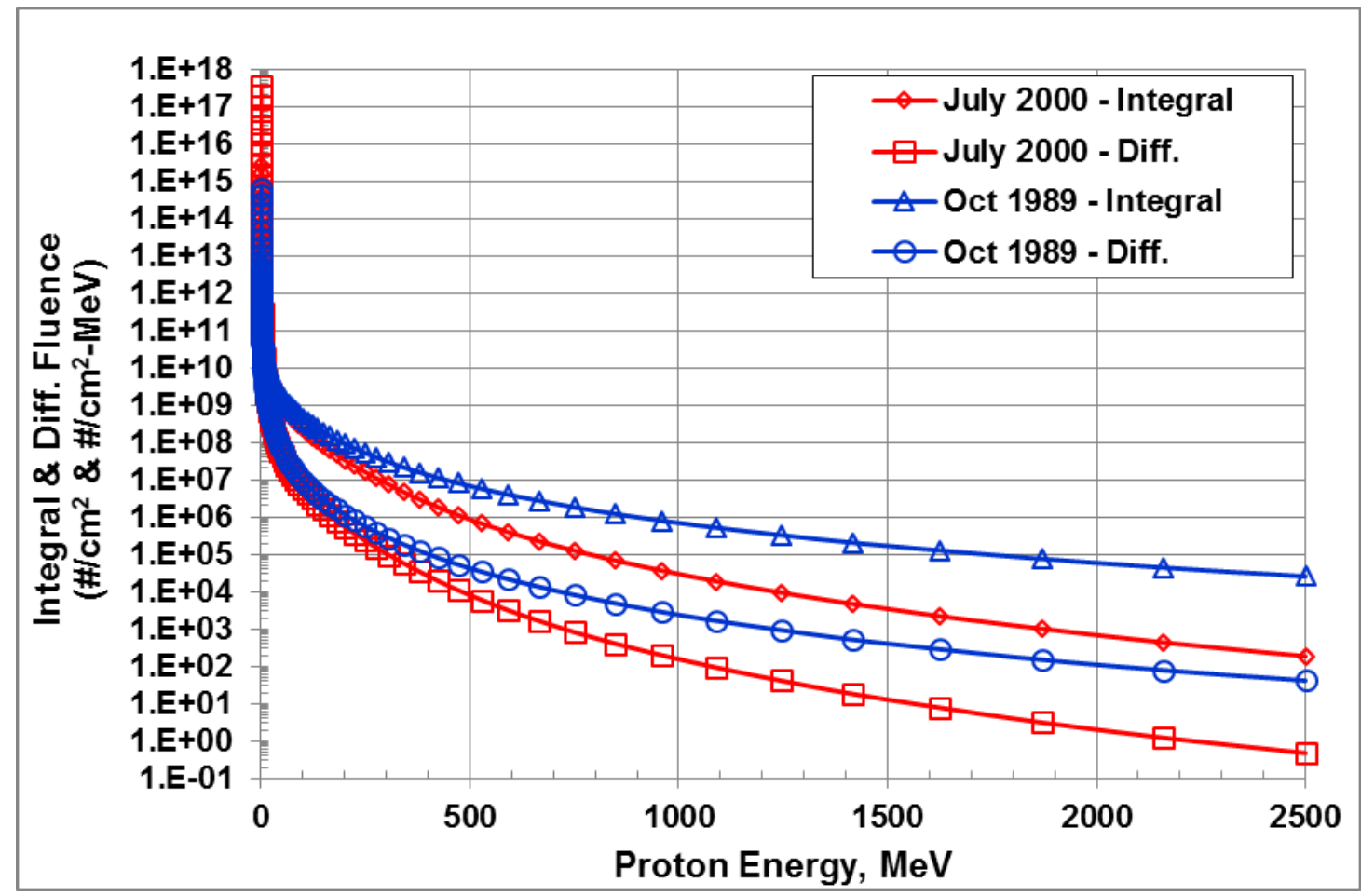

Figure 1: Integral and differential energy spectra for two extremely large SPEs that occurred on October 1989 and 14 July 2000. From 19-24 October 1989 there were three GLEs and an ESP, and the energy spectra shown in the plot is a combination of these four events.

Figure 2 is a plot of the GCR energy spectra for several elemental species for both solar minimum and solar maximum.

American Institute of Aeronautics and Astronautics 


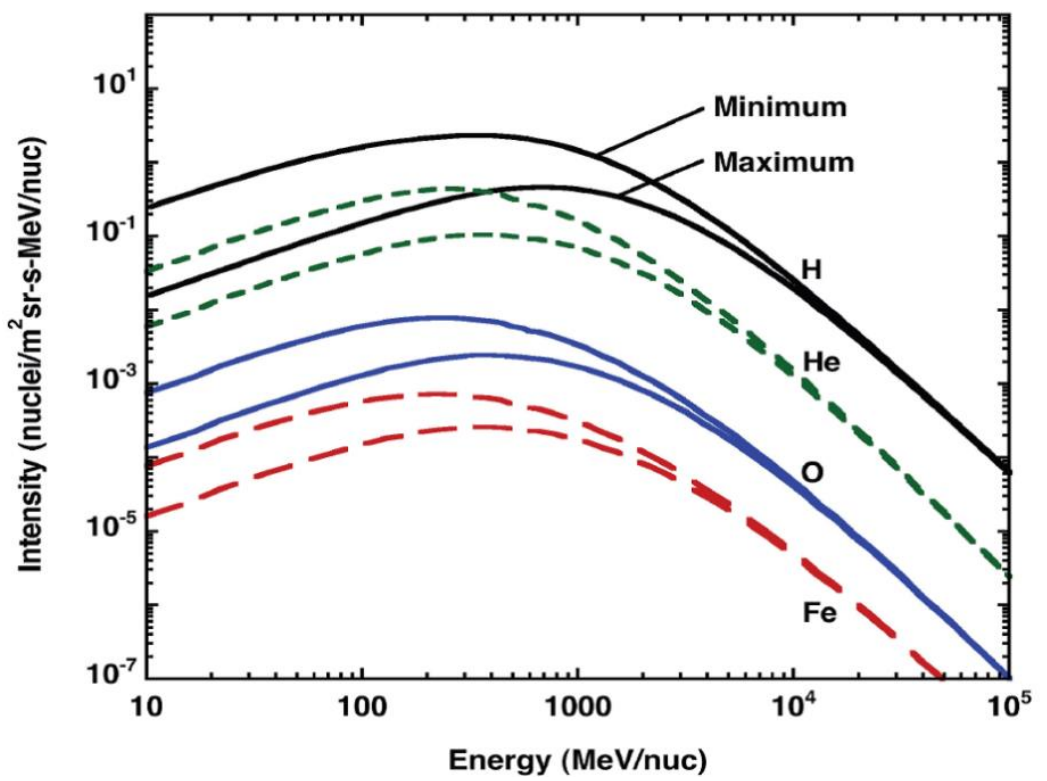

Figure 2: Differential fluence of several GCR elemental species $(\mathrm{H}, \mathrm{He}, \mathrm{O}$, and $\mathrm{Fe})$ at solar minimum and solar maximum conditions. ${ }^{4}$

In addition, GCR particles are extremely energetic, extending into the several GeV range (as does the GLEs), and it is these GCR particles that will produce a constant source of both primary and secondary radiation, mostly neutrons, exposures to the crew and onboard systems, including food and materials.

\section{Secondary radiation production and sources}

As was mentioned above, when these very energetic particles penetrate other materials (bulk shielding of the spacecraft, human body, and onboard systems, avionics, etc.) secondary particles, mostly neutrons, are produced due to nuclear reactions that occur.

We use the example of secondary neutrons produced in the Earth's atmosphere (albedo neutrons). As very high energy primary cosmic rays continuously arrive at the Earth, they interact with atoms in the Earth's atmosphere creating secondary particles. Of the possible secondary particles, high energy neutrons have been shown to be mainly responsible for causing single event upsets (SEUs) in memories and other devices in aircraft since the early 1990s.

For the neutrons, as well as all other secondary particles within the atmosphere, the variation of the particle flux with three parameters (altitude, latitude, and energy) is of primary importance for understanding variation of the single event effects (SEE) rate.

Altitude variation of atmospheric neutrons derives from competition between various production and removal processes that affect how the neutrons and the initiating cosmic rays interact with the atmosphere. The result of the neutron interaction with the atmosphere is a maximum in the flux at about 60,000 feet called the Pfotzer maximum, and an overall variation of the neutron flux (energy range of 1-10 MeV) with altitude (based on aircraft measurements). Figure 3 shows the neutron flux density as it varies with altitude. ${ }^{5}$

American Institute of Aeronautics and Astronautics 


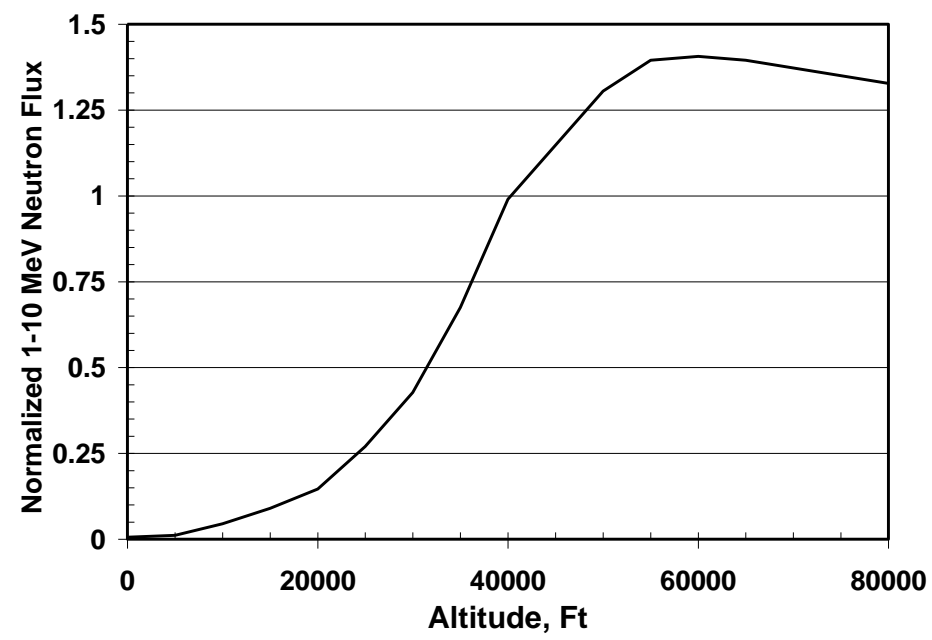

Figure 3: Simplified model of atmospheric neutron flux variation with altitude.

The latitude variation of the atmospheric neutrons is governed primarily by the Earth's magnetic field. The overall variation of the atmospheric neutron flux (energy range of 1-10 MeV) with latitude (based on aircraft measurements) is shown graphically in Figure 4.



Figure 4: Simplified model of atmospheric neutron flux variation with latitude.

There is an additional SEU rate in some devices contributed by the low energy neutrons (called thermal neutrons) that are produced in spacecraft and aircraft. While the high energy neutrons cause SEUs through interaction with the $\mathrm{Si}$ atoms, thermal neutrons cause SEUs through their interaction with boron-10 that is found in some microelectronics. Where possible, parts containing boron-10 or natural boron should be avoided. When assessing SEE rates, thermal neutron effects should be considered, when appropriate.

Thus, for deep space missions, GCR, SPEs, and secondary neutrons must be considered to assess the space radiation environment impact on the crew and onboard systems.

\section{B. Transport Codes}

FLUKA, short for FLUktuierende KAskade, is a Monte Carlo radiation transport code containing fully integrated physics capable of simulating the interaction of space radiation with material. The origins of FLUKA date back to the 1960 's, with the latest version able to simulate the propagation of a wide range of particles through virtually all materials and with applications to accelerator projects, shielding studies, cosmic rays studies, medical and 
radiobiology studies, and spacecraft electronics single event effects. For nuclear interactions relevant in this study, including neutrons, above $5 \mathrm{GeV} / \mathrm{n}$, FLUKA employs DPMJET-III (Dual Parton and Jets version 3) with a modified initialization procedure, and for energies between 0.1 and $5 \mathrm{GeV} / \mathrm{n}$, a modified RQMD (Relativistic Quantum Molecular Dynamics) model is used. For low energy nucleus interactions below $0.1 \mathrm{GeV} / \mathrm{n}$, the Boltzmann Master Equation is used. All models are followed by a generalized intra-nuclear cascade process followed by a preequilibrium stage and then an evaporation/fragmentation-fission stage. ${ }^{6-7}$

Neutrons below $20 \mathrm{MeV}$ are considered low-energy neutrons in FLUKA and are transported by a multigroup transport technique for computational considerations. Below $20 \mathrm{MeV}$, the neutrons are broken down into 260 energy groups, each approximately equal in logarithmic width, where both elastic and inelastic individual neutron interactions are tracked through group-to-group interaction probabilities. These probabilities are based on recent versions of the ENDF/B and JENDL-3 nuclear data files and contain data for up to 270 isotopes/materials at two different temperatures (some at more). The low energy neutron transport technique also includes effects such as Doppler broadening, gamma generation, energy deposition by kerma factors, residual nuclei production, secondary neutrons, and fission neutrons. ${ }^{6-7}$

The high charge and energy transport code, HZETRN2010, ${ }^{8-9}$ is a one dimensional, deterministic transport code developed at NASA Langley Research Center. The code uses a straight ahead approximation of the Boltzmann transport equation for charged and neutral particles, with a continuous slowing down approach. The advantages to using this code are that it has short run times and can be used to investigate any number of materials and configurations for radiation transport. This version of the code includes a bi-directional, coupled neutron transport model in its treatment of neutrons during slab transport and has been shown to have good agreement with Monte Carlo codes. ${ }^{9}$

\section{Method}

The CREME96 GCR model was used to generate both the HZETRN2010 and FLUKA input particle spectra data for this assessment. Solar maximum environment conditions and a low Earth orbit were selected to enable comparison with the available flight data. An altitude of $362.5 \mathrm{~km}$, orbit inclination of $51.6^{\circ}$ and quiet magnetic conditions were specified for the assessment. AP8MAX trapped protons were also included. To reduce the processing time needed for transport, seven species were selected to represent the input environment. These species are trapped and GCR protons, alphas, heavy ions of carbon, oxygen, magnesium, silicon, and iron. For the HZETRN2010 calculations, the CREME96 generated spectra were interpolated against the HZETRN2010 energy grid. The CREME96 GCR particle spectra are plotted in Figure 5.

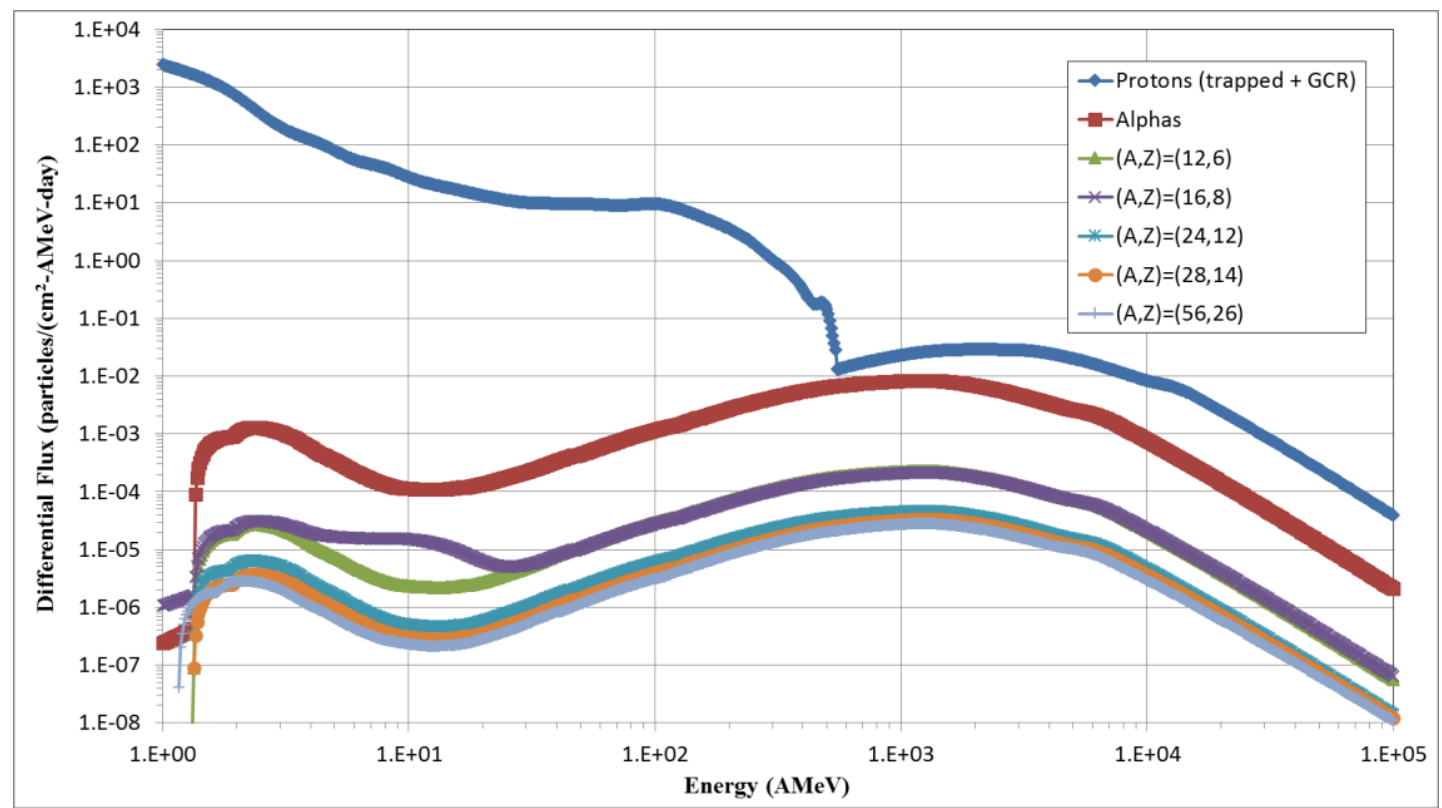

Figure 5: CREME96 GCR particle spectra used as the input environment for both the FLUKA and HZETRN2010 transport.

A range of shielding masses were selected for investigation to determine whether there are vast differences in the neutron production as a function of spacecraft thickness, as well as providing applicability of the data to a wide

American Institute of Aeronautics and Astronautics 
range of spacecraft types and missions. The shielding material selected is aluminum at the following thicknesses: 0.1 , $0.5,1,5,10,20,50,100,150$ and $200 \mathrm{~g} / \mathrm{cm}^{2}$.

The input environment generated by the CREME96 GCR model, along with the representative vehicle shielding thicknesses, were input into both FLUKA and HZETRN2010. The two codes were run in specific configurations to facilitate more accurate comparison between them.

For the purposes of this study, FLUKA can easily simulate low Earth orbit proton spectra, as well as galactic cosmic radiation, over the energy range of interest, including the peak flux of particles, and generate reliable neutron spectra as a function of spacecraft shielding. Differential spectra were sampled based on their cumulative probability distribution and input files were created for FLUKA. Each line in the input file contained the particle start position, direction cosines, and energy level. This file was combined with an input file containing details of the ion being transported, as well as the geometry for the simulation and definition of the desired output differential flux spectra. For the FLUKA simulation presented in this paper, the following number of primary ions were simulated (Table 1).

Table 1: Number of particles simulated.

\begin{tabular}{|c|c|c|c|c|c|c|c|c|c|c|}
\hline & $\begin{array}{c}\mathbf{0 . 1} \\
\mathbf{g} / \mathbf{c m} 2\end{array}$ & $\begin{array}{c}\mathbf{0 . 5} \\
\mathbf{g / c m} 2\end{array}$ & $\begin{array}{c}\mathbf{1 . 0} \\
\mathbf{g} / \mathbf{c m} 2\end{array}$ & $\begin{array}{c}\mathbf{5 . 0} \\
\mathbf{g} / \mathbf{c m} 2\end{array}$ & $\begin{array}{c}\mathbf{1 0} \\
\mathbf{g} / \mathbf{c m} 2\end{array}$ & $\begin{array}{c}\mathbf{2 0} \\
\mathbf{g} / \mathbf{c m} 2\end{array}$ & $\begin{array}{c}\mathbf{5 0} \\
\mathbf{g} / \mathbf{c m} 2\end{array}$ & $\begin{array}{c}\mathbf{1 0 0} \\
\mathbf{g} / \mathbf{c m} 2\end{array}$ & $\begin{array}{c}\mathbf{1 5 0} \\
\mathbf{g} / \mathbf{c m} 2\end{array}$ & $\begin{array}{c}\mathbf{2 0 0} \\
\mathbf{g} / \mathbf{c m} 2\end{array}$ \\
\hline $\mathbf{Z = 1}$ & $5.0 \mathrm{e} 9$ & $5.0 \mathrm{e} 9$ & $5.0 \mathrm{e} 9$ & $3.2 \mathrm{e} 9$ & $2.2 \mathrm{e} 9$ & $1.0 \mathrm{e} 9$ & $5.0 \mathrm{e} 8$ & $5.0 \mathrm{e} 8$ & $1.3 \mathrm{e} 9$ & $2.5 \mathrm{e} 8$ \\
\hline $\mathbf{Z = 2}$ & $4.9 \mathrm{e} 9$ & $2.8 \mathrm{e} 9$ & $9.6 \mathrm{e} 8$ & $4.0 \mathrm{e} 8$ & $2.0 \mathrm{e} 8$ & $9.8 \mathrm{e} 7$ & $5.0 \mathrm{e} 7$ & $4.9 \mathrm{e} 7$ & $2.5 \mathrm{e} 7$ & $2.5 \mathrm{e} 7$ \\
\hline $\mathbf{Z = 6}$ & $5.9 \mathrm{e} 8$ & $5.7 \mathrm{e} 8$ & $2.9 \mathrm{e} 8$ & $5.0 \mathrm{e} 7$ & $2.9 \mathrm{e} 7$ & $1.5 \mathrm{e} 7$ & $2.4 \mathrm{e} 7$ & $2.7 \mathrm{e} 7$ & $2.4 \mathrm{e} 7$ & $2.5 \mathrm{e} 7$ \\
\hline $\mathbf{Z = 8}$ & $8.9 \mathrm{e} 8$ & $3.0 \mathrm{e} 8$ & $1.2 \mathrm{e} 8$ & $3.0 \mathrm{e} 7$ & $1.8 \mathrm{e} 7$ & $9.0 \mathrm{e} 6$ & $2.4 \mathrm{e} 7$ & $7.4 \mathrm{e} 6$ & $2.4 \mathrm{e} 7$ & $2.5 \mathrm{e} 7$ \\
\hline $\mathbf{Z = 1 2}$ & $4.7 \mathrm{e} 8$ & $1.2 \mathrm{e} 8$ & $5.0 \mathrm{e} 7$ & $1.6 \mathrm{e} 7$ & $5.0 \mathrm{e} 6$ & $4.8 \mathrm{e} 6$ & $4.9 \mathrm{e} 6$ & $7.3 \mathrm{e} 6$ & $1.2 \mathrm{e} 7$ & $5.0 \mathrm{e} 6$ \\
\hline $\mathbf{Z = 1 4}$ & $3.7 \mathrm{e} 8$ & $1.1 \mathrm{e} 8$ & $5.0 \mathrm{e} 8$ & $1.3 \mathrm{e} 7$ & $5.0 \mathrm{e} 6$ & $3.9 \mathrm{e} 6$ & $4.1 \mathrm{e} 6$ & $7.3 \mathrm{e} 6$ & $1.2 \mathrm{e} 7$ & $5.0 \mathrm{e} 6$ \\
\hline $\mathbf{Z = 2 6}$ & $1.2 \mathrm{e} 8$ & $3.2 \mathrm{e} 7$ & $1.8 \mathrm{e} 7$ & $4.2 \mathrm{e} 6$ & $2.4 \mathrm{e} 6$ & $1.2 \mathrm{e} 6$ & $2.4 \mathrm{e} 6$ & $1.0 \mathrm{e} 6$ & $1.2 \mathrm{e} 6$ & $2.5 \mathrm{e} 6$ \\
\hline
\end{tabular}

The simulations were performed on several clusters at various times, each consisting of several hundred nodes up to 1,000 nodes. Generally, the thick shielding runs were run on the cluster with the most available nodes because of the length of time required for completion. The simulations were performed with the PRECISIOn defaults in FLUKA, and double differential yields weighted by $1 / \cos ($ theta), where theta is the angle of the ion crossing the surface with respect to the normal, were obtained with respect to energy and charge. The charge was integrated out leaving output differential spectra, i.e. number of ions $/ \mathrm{cm}^{2} / \mathrm{GeV} / \mathrm{n} / \mathrm{sec}$ in each energy bin. Because the native output units of Monte Carlo codes are normalized per primary simulated, the output spectra were then normalized to the space level based on the total number of particles for each input spectrum. To facilitate comparison with HZETRN2010, the FLUKA results were interpolated against the HZETRN2010 energy grid.

For HZETRN2010, the CREME96 GCR input environment is considered a user defined environment. To run properly, it was formatted to fit the HZETRN2010 GCR environments definition database, which includes columns for all $59 \mathrm{GCR}$ isotopes. Each material thickness was run separately due to the forward and backward treatment of the neutron transport to yield a total of ten runs. The transported neutron flux results were compiled for comparison with FLUKA.

The On-Line Tool for the Assessment of Radiation in Space (OLTARIS ${ }^{10}$ ) was used to provide neutron albedo information in low Earth orbit during solar maximum conditions. The neutron albedo model in OLTARIS is a parametric fit of data gathered by NASA Langley Research Center. ${ }^{10,11}$ The altitude and inclination were specified to $362.5 \mathrm{~km}$ and $51.6^{\circ}$, respectively, to remain consistent with the CREME96 GCR input environment used during transport. This neutron albedo environment was transported through the specified thicknesses mentioned above and provided a resulting neutron flux. To accurately compare the transported data with measured flight data, the transported neutron flux resulting from GCR, trapped protons, and albedo need to be combined. Thus, the output neutron flux resulting from albedo was then interpolated against the HZETRN2010 energy grid to enable summation with the transport results from HZETRN2010 and FLUKA. The combined results were then compared with flight data.

\section{Results}

\section{A. HZETRN2010 and FLUKA Comparison}

The FLUKA neutron fluxes behind various levels of aluminum shielding are plotted along with the HZETRN2010 results in Figure 6 and Figure 7 below. Flux error bars are plotted on the FLUKA data at each decade in energy; however, they appear to be no larger than the width of the plot line. The simulations show fairly good agreement in 
overall shape and magnitude, with the FLUKA simulation being slightly higher in magnitude. However, at high energies (several hundred $\mathrm{MeV} / \mathrm{n}$ ), the two data sets appear to match well at all shielding thicknesses. The fluctuations in the FLUKA data at low energy are attributed to poor statistics. The inflection points owing to the trapped proton environment appear to be more distinct in the HZETRN2010 data and more smeared out in the FLUKA data.
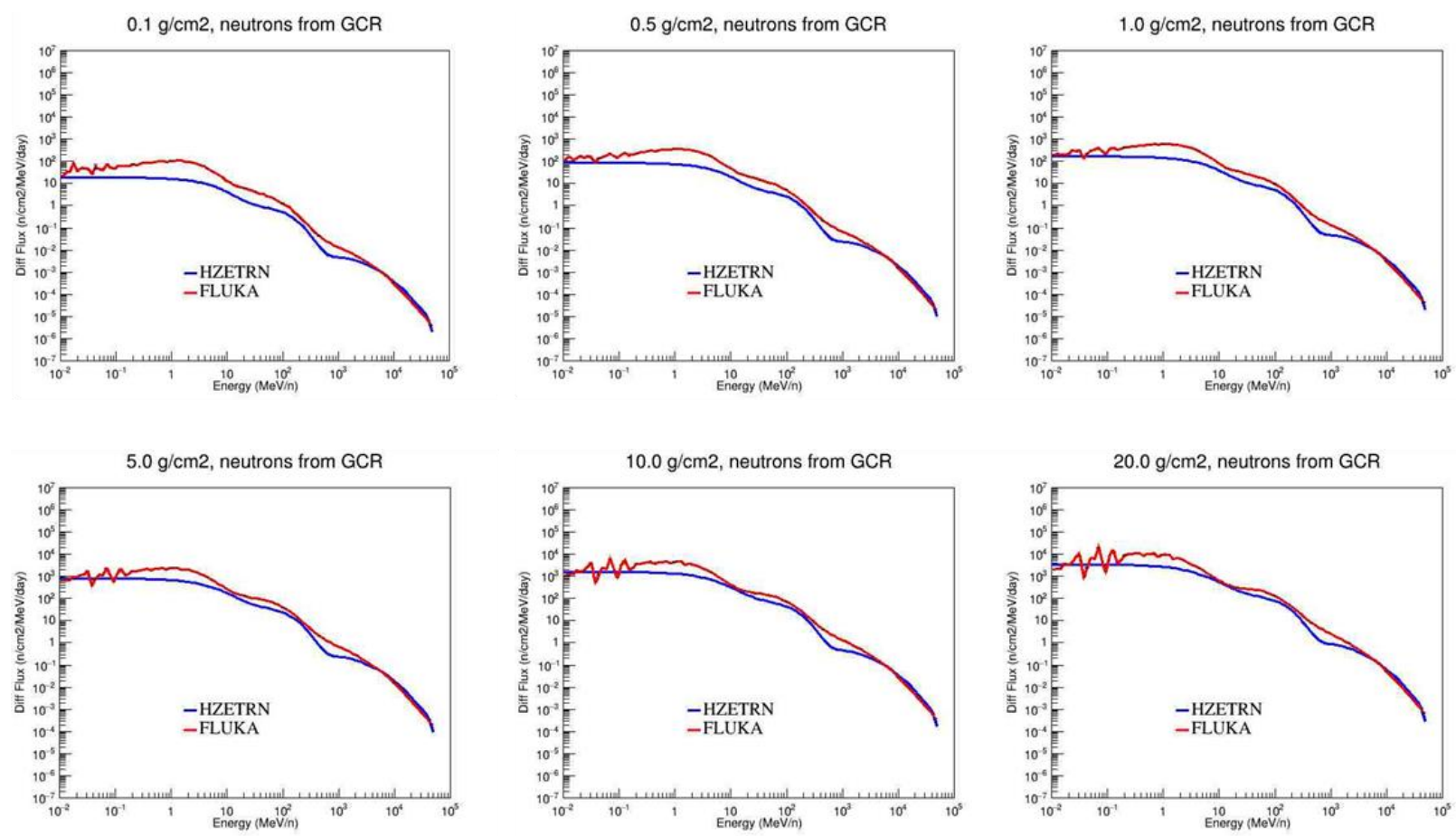

Figure 6: Comparison of HZETRN2010 and FLUKA for thicknesses ranging from 0.1 to $20 \mathrm{~g} / \mathrm{cm}^{2}$.
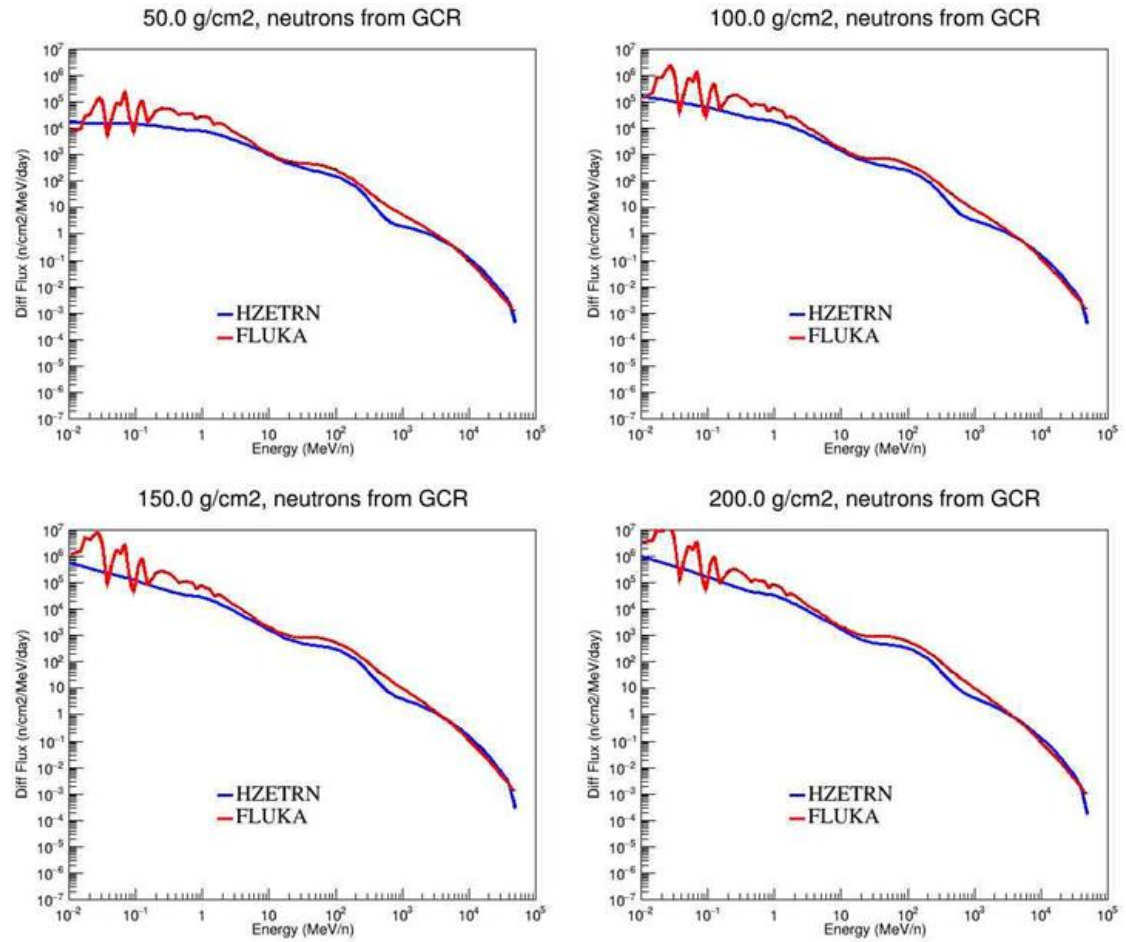

Figure 7: Comparison of HZETRN2010 and FLUKA for thicknesses ranging from 50 to $200 \mathrm{~g} / \mathrm{cm}^{2}$.

American Institute of Aeronautics and Astronautics 
To compare the data in another way, each of the data sets plotted in Figure 6 and Figure 7 were integrated over the energy levels using the trapezoidal rule (Equation 1). The integrated data was then plotted against thickness to better understand how the neutron flux output changed with increased shielding mass (Figure 8).

$$
\sum\left(X_{i}-X_{i-1}\right)\left(\frac{f\left(X_{i-1}\right)+f\left(X_{i}\right)}{2}\right)
$$

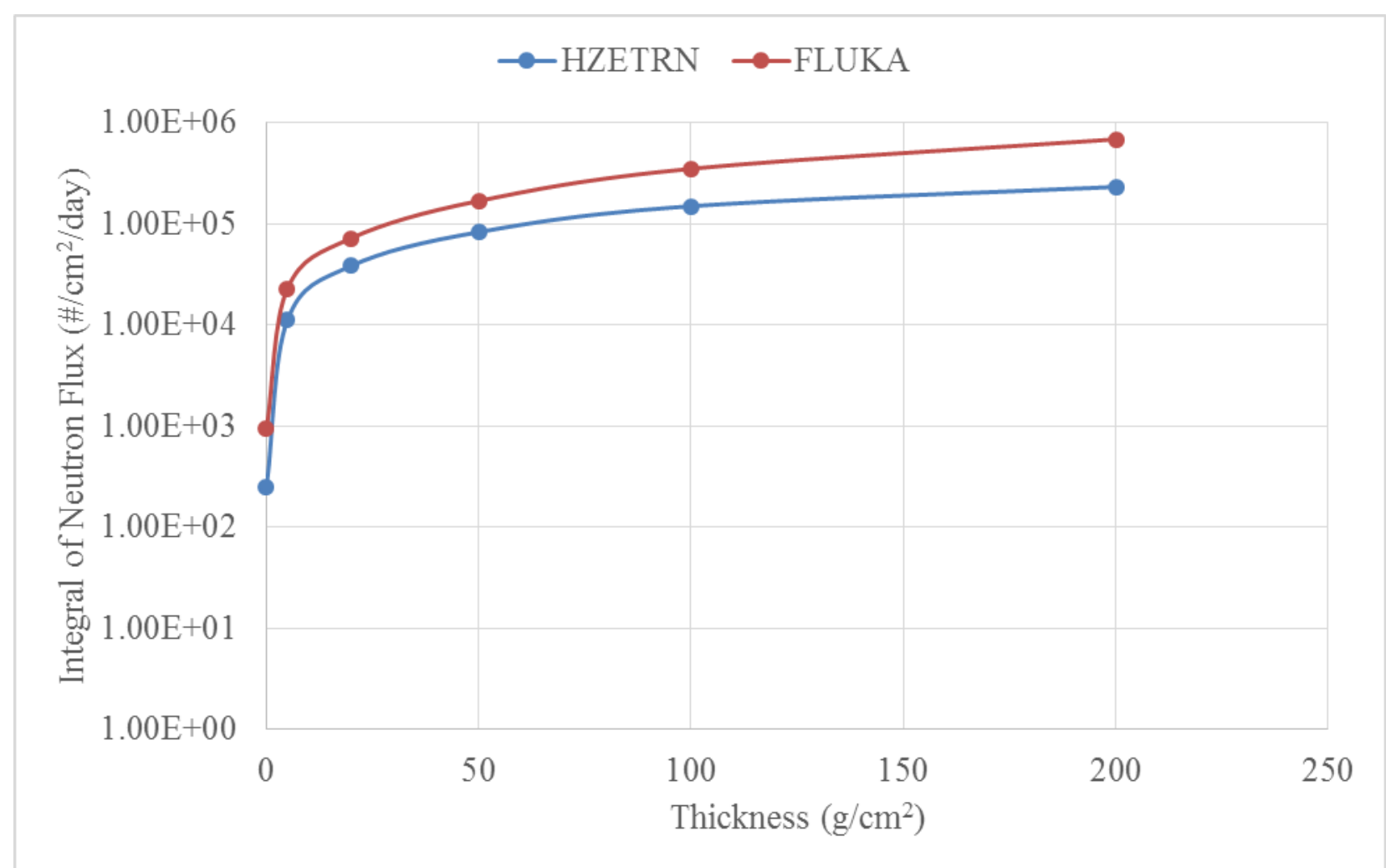

Figure 8: Integrated neutron flux of both HZETRN2010 and FLUKA results plotted against thickness of shielding mass.

\section{B. Simulation Data Compared with Flight Data}

There were four sets of flight data extracted for comparison with the FLUKA and HZETRN2010 results. All four sets of measurements were made during solar maximum conditions from 1989 to 2001 . The first two sets of data ${ }^{12}$ were from space shuttle missions. STS-28 occurred on August 8, 1989 during solar cycle 22, and the orbit was located at $302 \mathrm{~km}$ with a $57^{\circ}$ inclination. STS-36 occurred on February 28, 1990, also during solar cycle 22, with an orbit at $246 \mathrm{~km}$ and inclination of $62^{\circ}$. The neutron data acquired during these flights was via a Bonner Ball neutron detector. The locations of the detector were not specified in the paper, and the shielding mass in front of the detector is unknown.

The third set of flight data ${ }^{13}$ came from the Mir space station during the 1990-1992 timeframe, also during solar cycle 22. Mir was located at an altitude between $354 \mathrm{~km}$ and $374 \mathrm{~km}$ with an inclination of $51.6^{\circ}$. The detector used during this experiment was a fission foil detector. The location of the detector is unknown, but the shielding thickness in front of the detector is estimated at $40 \mathrm{~g} / \mathrm{cm}^{2}$.

The final set of flight data ${ }^{14}$ is an average of neutron flux data collected during a mission aboard the International Space Station (ISS). This data was collected during solar cycle 23 and was collected at two locations within the ISS. The first data collection occurred on March 23, 2001 and was located at the US lab rack 1P1. The second data collection occurred on November 14, 2001 at the US lab rack 1D3. The data was measured using a Bonner Ball neutron detector and the shielding thickness in front of the detector ranged from $20-50 \mathrm{~g} / \mathrm{cm}^{2}$.

Given that all of the extracted flight data was collected behind a shielding mass in the range of $20-50 \mathrm{~g} / \mathrm{cm}^{2}$, we focused our comparison of HZETRN2010 and FLUKA on $50 \mathrm{~g} / \mathrm{cm}^{2}$ shielding. The results of the comparison are shown in Figure 9.

American Institute of Aeronautics and Astronautics 


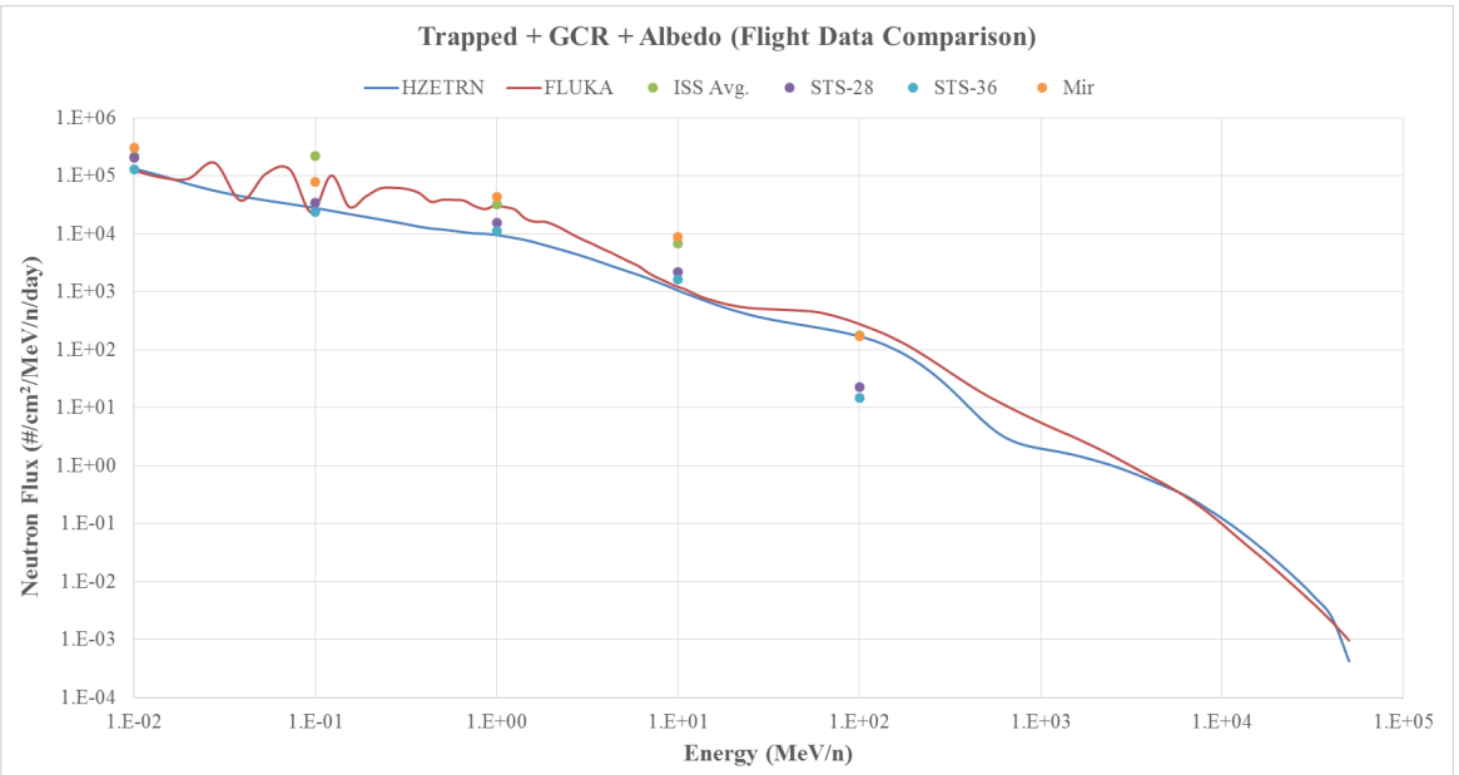

Figure 9: Comparison of four sets of flight data with FLUKA and HZETRN2010 simulation results at $50 \mathrm{~g} / \mathrm{cm}^{2}$ thickness.

\section{Discussion}

In this study, HZETRN2010 and FLUKA results show similar agreement, and the differences would result in FLUKA predicting slightly higher rates for neutron related effects, such as dose from neutrons or displacement damage dose for materials. The comparison performed in this study doesn't show as good agreement as in previous comparisons, which have generally been done for proton only environments, such as solar particle events or GCR environments separately, but not combined. ${ }^{15}$ This same prior work uses different sampling techniques for these two environment regimes because each environment spans different energy scales. Source energy biasing is desired for low energy proton spectra, such as that from solar particle events or trapped radiation, to improve neutron statistics, something that was not done in this study because the trapped protons were combined with the galactic cosmic ray proton spectra. Although not discussed in this paper, this simulation shows much better agreement in predicted proton fluxes across all thicknesses. Therefore, in conclusion for the HZETRN2010 and FLUKA comparison, the two codes agree well enough to make comparisons to the flight data.

In studying the simulation data, specifically Figure 8, it is apparent that with increasing shielding mass, there is an increase in the neutron flux. This increase is quite dramatic at lower thicknesses and then begins to decrease in slope at higher thicknesses. This observation could be cause for concern as vehicle sizes increase for human missions to deep space and to Mars.

The flight data was compared against the FLUKA and HZETRN2010 simulation results at $50 \mathrm{~g} / \mathrm{cm}^{2}$ thickness. From $0.01 \mathrm{MeV}$ to $100 \mathrm{MeV}$, the simulation and collated flight data agree very well. The simulation results extend out to $50 \mathrm{GeV}$, but the flight data only extends to $100 \mathrm{MeV}$. Therefore, it is unknown whether that agreement will continue at higher energies. The space shuttle neutron flux was consistently lower than the ISS and Mir space station fluxes. Given that the locations of the neutron detectors and thus the shielding thicknesses in front of the detectors was not well known in all of the data, it is possible that this difference in neutron flux is a result of different shielding thicknesses between the space shuttle and the two space stations. Furthermore, there is some uncertainty as to how well this flight data agrees to the simulation data without knowing the approximate shielding thicknesses in front of the detectors. As Figure 8 shows, a contributor to the increase in neutron flux is the shielding mass thickness of the vehicle. Thus, further research into these neutron experiments to verify the shielding information is necessary. Additionally, other experiments with more precise information on shielding mass, larger ranges of shielding mass and larger ranges of energy are necessary to verify completely the results from the simulation.

American Institute of Aeronautics and Astronautics 


\section{Conclusion}

The results of this study show that there is good agreement between FLUKA and HZETRN2010 in the neutron fluxes. Thus, future neutron studies can use HZETRN2010 to perform quick analyses of simple shielding geometries to determine neutron yields and understand effects of neutrons. As a vehicle design gets more refined and requires complex analysis, FLUKA may be a better tool to understand the full neutron environment produced by the vehicle.

Furthermore, comparing the simulation results to neutron flight data showed good agreement at a vehicle thickness of $50 \mathrm{~g} / \mathrm{cm}^{2}$. Additional work is needed to further validate the simulation data at greater energies and different thicknesses.

Finally, our observations showed an overall increase in the neutron flux with increasing thickness of the vehicle. This result may be a cause for concern as vehicle sizes increase for human missions to deep space and Mars. Future work needs to be completed to validate the simulation data at these larger thicknesses and the implications to crew, avionics, and materials due to the increase in neutron flux needs to be well understood.

This work is a first step at understanding the neutron environment resulting from primary radiation interactions with the vehicle shielding mass. Future work will also encompass the neutron albedo environment from the surface of Mars as another source of neutron radiation. Better understanding the surface albedo environment, as well as the interactions of that surface albedo with the vehicle shielding mass to produce a secondary neutron environment, is not well understood. Thus, further analysis in this area, as well as investigating the implications of the secondary neutron environment from the Martian surface will be important to future Mars missions.

\section{References}

1NASA, "Human Exploration of Mars Design Reference Architecture 5.0," NASA-SP-2009-566, 2009.

${ }^{2}$ Toups, L., Brown, K., Hoffman, S.J., "Transportation-driven Mars Surface Operations Supporting an Evolvable Mars Campaign," Proceedings of IEEE Aersopace, Big Sky, MT, 2015.

${ }^{3}$ Tylka, A., Dietrich, W., and Atwell, W., "Band Function Representations of Solar Proton Spectra in Ground-Level Events," $38^{\text {th }}$ Scientific Assembly of the Committee on Space Research (COSPAR), 2010.

${ }^{4}$ Badwhar, G.D., Cucinotta, F.A., and O’Neill, P.M., "An analysis of interplanetary space radiation exposure for various solar cycles," Radiation Research, 138(2), 201-208, 1994.

${ }^{5}$ Normand, E. "Correlation of in flight neutron dosimeter and SEU measurements with atmospheric neutron model," Nuclear Science, IEEE Transactions, 48(6), 1996 - 2003, 2001.

${ }^{6}$ Battistoni, G., Muraro, S., Sala P., Cerutti, F., Ferrari, A., Roesler, S., Fassò, A., and Ranft, J., "The FLUKA Code: Description and Benchmarking", Proceedings of the Hadronic Shower Simulation Workshop 2006, Fermilab 6--8 September 2006, M.Albrow, R. Raja eds., AIP Conference Proceeding 896, 31-49, 2007.

${ }^{7}$ Fassò, A., Ferrari, A., Ranft, J., Sala P., "FLUKA: A Multi-particle Transport Code", CERN-2005-10, INFN/TC_05/11, SLAC-R-773, 2005.

${ }^{8}$ Slaba, T.C., Blattnig, S.R., Badavi, F.F., "Faster and more accurate transport procedures for HZETRN," Journal of Computational Physics, 229, 9397-9417, 2010a.

${ }^{9}$ Slaba, T.C., Blattnig, S.R., Aghara, S.K., Townsend, L.W., Handler, T., Gabriel, T.A., Pinsky, L.S., Reddell, B., “Coupled Neutron Transport for HZETRN," Radiation Measurements, 45, 173-182, $2010 \mathrm{~b}$.

${ }^{10}$ Singleterry, R.C., Blattnig, S.R., Clowdsley, M.S., Qualls, G.D., Sandridge, C.A., Simonsens, L.C., Norbury, J.W., Slaba, T.C., Walker, S.A., Badavi, F.F., Spangler, J.L., Aumann, A.R., Zapp, E.N., Rutledge, R.D., Lee, K.T., Norman, R.B., "OLTARIS: On-Line Tool for the Assessment of Radiation in Space," NASA TP-2010-216722, 2010.

${ }^{11}$ Wilson, J.W., et al., "Cosmic Ray Neutron Albedo Dose in Low Earth Orbit," Health Physics, 57, 665-668, 1989.

${ }^{12}$ Atwell, W., Badhwar, G., "Passive Neutron Measurements: Some early Mir/Space Shuttle results," Proceedings of the 45th International Conference on Environmental Systems, Bellevue, WA, 2015.

${ }^{13}$ Getselev, I., Rumin, S., Sobolevsky, N., Ufimtsev, M., Podzolko, M., "Absorbed dose of secondary neutron from galactic cosmic rays inside the International Space Station," Advances in Space Research, 34, 1429-1432, 2004.

${ }^{14}$ Koshiishi, H., Matsumoto, H., Chishiki, A., Goka, T., Omodaka T., "Evaluation of the neutron radiation environment inside the International Space Station based on the Bonner Ball Neutron Detector Experiment," Radiation Measurements, 42, 1510-1520, 2007.

${ }^{15}$ Wilson, J., Slaba, T., Badavi, F.F., Reddell, B., and Bahadori, A., “3DHZETRN: Shielded ICRU spherical phantom”, Life Sciences in Space Research, 4, 46-61, 2015.

American Institute of Aeronautics and Astronautics 